\title{
S.O.A. ¿Una Manifestación Clínico-Patológica de Diferentes Estados Hiperandrogénicos?
}

\author{
Gustavo Gómez T., M.D. * \\ Enrique Herrera C., M.D. * * \\ Raúi M. Escobar N., M.D. ** \\ Eduardo Romero V., M.D. **
}

\section{INTRODUCCION}

En un período de cinco años se han diagnosticado y tratado 194 pacientes con síndrome de ovario androgénico en la clínica de Endocrinología e Infertilidad del Hospital Universitario del Valle. Sus características clínicas, determinaciones hormonales, evaluación y respuesta al tratamiento, son manifestaciones de los diferentes estados clínicos y patológi$\cos$ de una entidad que tiene como manifestación persistente un estado hiperandrogénico.

\section{MATERIALES, METODOS, PACIENTES}

En las pacientes que asisten a la Consulta Externa de Ginecologia y en las que se determina por historia clínica y examen físico que: a) está en anovulación, b) es hirsuta y/o obesa, c) está en

* Profesor Auxiliar. Departamento de Obstetricia y Ginecología. Coordinador Clínica Endocrinología e Infertilidad. Hospital Universitario del Valle. Cali - Colombia.

** Residente Tercer Año. Departamento de Obstetricia y Ginecología. Hospital Universitario del Valle. Cali - Colombia. amenorrea secundaria con prueba de progesterona positiva; a partir de la fecha en que estas pacientes consultaron y en una forma evolutiva a través de los cinco años del estudio se les realizó, además de un completo examen físico, determinaciones de FSH - LH - PRL y T por radioinmunoensayo, en el laboratorio central de Endocrinología del H.U.V., por técnicas ya establecidas. Las pacientes que consultan además por infertilidad en algún momento del estudio se les realizó una laparoscopia diagnóstica.

\section{RESULTADOS}

Oligomenorrea, amenorrea, hirsutismo e infertilidad en su orden, fueron los signos y síntomas mas frecuentes (Cuadro No. 1). Los grupos de edad donde principalmente se detectó fue entre 20-35 años $(48.8 \%)$ como una cifra importante en la mujer joven, menor de 20 años (28. $8 \%$ ). Después de los 35 años no tuvimos pacientes con diagnóstico de SOA.

En 165 pacientes se hicieron determinaciones de Testosterona total (Cuadro No. 2) y en el $64.2 \%$, se encontraron valores superiores a $\mathbf{5 0} \mathbf{n g} / \mathbf{d l}$., datos 
Cuadro No. 1

194 PACIENTES CON S.O.A. SINTOMATOLOGIA

\begin{tabular}{|lrr|}
\hline & $\mathbf{n}$ & $\%$ \\
\hline OLIGOMENORREA & 156 & 80.4 \\
AMENORREA & 142 & 73.1 \\
HIRSUTISMO & 113 & 58.2 \\
INFERTILIDAD & 75 & 38.6 \\
OBESIDAD & 74 & 38.1 \\
GALACTORREA & 40 & 20.6 \\
DOLOR PELVICU & 24 & 12.3 \\
METRORRAGIA & 10 & 5.1 \\
VIRILIZACION & 10 & 5.1 \\
\hline
\end{tabular}

Cuadro No. 2

TESTOSTERONA EN 165 PACIENTES CON S.O.A.

\begin{tabular}{|cccc|}
\hline & $\mathbf{n g ~ / ~} \mathbf{m l}$ & $\mathbf{n}$ & $\%$ \\
\hline 1 & $20-30$ & 11 & 6.6 \\
2 & $30-40$ & 15 & 9.0 \\
3 & $40-50$ & 33 & 20.0 \\
4 & $50-60$ & 37 & 22.4 \\
5 & $>-60$ & 69 & 41.8 \\
\hline
\end{tabular}

también consistentes con anteriores publicaciones (16). En 116 pacientes se realizaron determinaciones de PRL y el 34. $4 \%$ ten ían valores mayores de $20 \mathrm{ng} / \mathrm{nl}$ con un $7.7 \%$ (9 pacientes) con valores entre 50-80 $\mathrm{ng} / \mathrm{nl}$, sin evidencia de adenoma hipofisiario (Cuadro No. 3).

Se hicieron determinaciones de $\mathrm{FSH} y$ $\mathrm{LH}$ y se calculó la relación $\mathrm{LH} / \mathrm{FSH}$ en 45 pacientes encontrándose que el $60 \%$ ten ían una relación 3:1, ó mayor. (Tabla No. 2)

Cuadro No. 3

PROLACTINA EN 116 PACIENTES CON S.O.A.

\begin{tabular}{|ccc|}
\hline $\mathrm{ng} / \mathrm{ml}$ & No. & $\%$ \\
\hline$<20$ & 76 & 65.5 \\
$20-50$ & 31 & 26.7 \\
$50-80$ & 9 & 7.7 \\
\hline
\end{tabular}

En 76 pacientes hicimos laparoscopia diagnóstica. Las clasificamos así: a) "características" de SOA $(n=48)$, cuando macroscópicamente los ovarios eran: aumentados de tamaño, aperlados, con cápsula engrosada, generalmente sin signos de ovulación; b) "dudosos" para SOA $(n=21)$ : ovarios de tamaño normal, blancos aperlados sin signos de ovulación y cápsula engrosada; c) "negativos" para SOA $(n=7)$ : ovarios pequeños, lobulados, sin signos de ovulación, quistes foliculares subcapsulares. El $92 \%$ de las pacientes presentaban hirsutismo y algunos de los signos o síntomas de oligomenorrea, infertilidad y obesidad.

En 18 de las pacientes con apariencia laparoscópica "caracter ística" de SOA en que se realizó concomitantemente relación LH/FSH y T se encontró indistintamente $\mathrm{T} \uparrow \uparrow$ y/o relación $\mathrm{LH} / \mathrm{FSH}>3.0$ (Cuadro No. 4). Del segundo grupo de 21 pacientes con apariencia macroscópica "dudosa" para SOA, en 8 se realizaron concomitantemente LH/FSH y T y también se encontró indistintamente $\mathrm{T}$ y/o LH/FSH aumentados (Cuadro No. 5). De las 7 pacientes del tercer grupo sólo 3 ten ían ambas determinaciones y en 2 los valores de $\mathrm{T}$ estaban aumentados y $\mathrm{LH}$ : FSH menos de 3 y el restante tenían ambos valores bajos (Cuadro No. 6). 
Cuadro No. 4

LH/FSH Y T EN 18 DE 46 OVARIOS

"CARACTERISTICOS" DE S.O.A.

\begin{tabular}{|ccc|}
\hline LH/FSH & & Hirs. \\
\hline 5.2 & -3 & + \\
4.5 & -5 & + \\
4.4 & -5 & - \\
7.0 & -4 & + \\
1.8 & -5 & + \\
4.1 & -2 & - \\
4.1 & -2 & + \\
2.6 & -5 & + \\
3.9 & -5 & - \\
2.5 & -4 & + \\
3.5 & -3 & - \\
3.1 & -3 & + \\
3.2 & -5 & + \\
3.1 & -2 & + \\
3.0 & -1 & + \\
3.0 & -5 & + \\
3.0 & -5 & + \\
3.0 & -5 & + \\
\hline
\end{tabular}

Cuadro No. 5

LH/FSH Y T EN 8 DE 21 PACIENTES CON OVARIOS "DUDOSOS" PARA S.O.A.

\begin{tabular}{|ccc|}
\hline LH/FSH & \multicolumn{1}{c}{ T } & Hirs. \\
\hline 4.5 & 4 & - \\
4.1 & -1 & + \\
1.1 & -5 & + \\
3.0 & -5 & - \\
3.0 & -1 & + \\
3.0 & -2 & + \\
4.8 & -2 & - \\
2.0 & -5 & + \\
\hline
\end{tabular}

Cuadro No. 6

LH/FSH Y T EN 3 DE 7 PACIENTES CON OVARIOS "NEGATIVOS" PARA S.O.A.

\begin{tabular}{|ccc|}
\hline LH/FSH & T & Hirs. \\
\hline 3.8 & -4 & \\
4.3 & -5 & \\
1.1 & -3 & \\
\hline
\end{tabular}

Tabla No. 1

TRATAMIENTO EN 194 PACIENTES CON S.O.A.

\begin{tabular}{|lcccc|}
\hline CLOMIFENO & 68 & 42.6 & 70.6 & 33.8 \\
FERTODUR & 23 & 26.0 & 34.7 & 4.3 \\
PROVERA & 55 & - & - & - \\
BR. ERGOCRIPTINA & 28 & 50.0 & 53.5 & 14 \\
CUÑA DE OVARIOS & 12 & - & 66.6 & \\
OVULEN & 22 & 73.0 & 80.7 & 26.8 \\
NINGUNO & 29 & - & - & - \\
\hline
\end{tabular}

Tabla No. 2

RELACION LH/FSH EN 45 PACIENTES CON S.O.A.

\begin{tabular}{|ccc|}
\hline LH/FSH & $n$ & $\%$ \\
\hline 1 & 9 & 20 \\
2 & 9 & 20 \\
3 & 11 & 24.4 \\
4 & 16 & 35.5 \\
\hline
\end{tabular}

TRATAMIENTO

El tratamiento fue diferente de acuerdo: a) deseo de embarazo, b) regularización de los ciclos, c) si estaba orientado a disminuír el hirsutismo y d) cuando había niveles altos de PRL (Tabla No. 1). 
En el primer caso se utilizó específicamente inductores de ovulación tipo clomifeno (clomid-omifin), o en su defecto Ciclofenil (Fertodur). Con Clomifeno tenemos $23 / 60$ embarazos $(38.3 \%)$ de los cuales 7 fueron a término, 7 prematuros, 5 abortos y 4 no regresaron a control.

Debido a que algunas parejas presentaban otros factores comprometidos cuando se administró Clomifeno $(n=7)$ el $\%$ de embarazo corregida en ese aspecto sería el $43.3 \%$. Los otros tipos de tratamiento en cuanto a regularización de los ciclos y porcentaje de embarazo se muestra en la Tabla No. 1.

\section{COMENTARIOS}

Desde 1935, cuando Stein y Leventhal describen el sindrome de obesidad, hirsutismo, infertilidad, ovarios poliquísticos e hiperticosis ovárica sin cuerpo lúteo (1), han aparecido periódicamente y de una manera creciente otras entidades que reunen algunas características del sindrome original descrito en 1935 y que incluye la poliquistosis ovárica (6), el hirsutismo idiopático (2), hiperticosis ovárica $(7,8)$ y anovulación hipotalámica crónica (9) como entidades separadas o en forma combinada en los cuales hay un factor común que es el hiperandrogenismo primario o secundario del ovario. Sin embargo en los diferentes sindromes descritos, el resultado final es, signos fenotípicos de hiperandrogenismo (acné, hirsutismo), como en el $58 \%$ de nuestras pacientes; 0 acciones hormonales del mismo, como es la anovulación y/o amenorrea $80.4 \%$ y $73.1 \%$ respectivamente. En el $64.2 \%$ de nuestras pacientes se encontraron niveles de $\mathrm{T}$ por encima de lo normal $(50 \mathrm{ng} / \mathrm{ml})$. Sin embargo sabemos que lo que medimos es $T$ total y en casos de "poliquistosis ovárica" e hirsutismo idiopático se han encontrado niveles de $T$ total normal, pero la fracción libre está elevada en todos los casos. Es la T libre la que se considera activa a nivel del órgano blanco (10), y esto sugiere que el estado hiperandrogénico es constante.

Las características morfológicas en el S.O.A. son: agrandamiento de ambos ovarios, cápsula lisa y engrosada. Esta aparenta ser avascular y al corte se encuentran numerosos quistes foliculares subcapsulares. La exposición continua de los ovarios a un ambiente hiperandrogénico conduce a que la mayoría de folículos antrales terminen en atresia (6). Estos continúan sintetizando androstendiona, pero pierden su actividad de aromatosa y por lo tanto disminuye su capacidad de sintetizar estrógenos. Aparentemente esta capacidad no puede recobrarse y se asocia con pérdida de la capacidad de dividirse las células de la granulosa. Por otra parte el proceso de atresia se incrementa, debido a un aumento relativo de andrógenos que antagoniza con el desarrollo folicular la cual es estrógeno dependiente (12). Esto explica el desarrollo de las múltiples quistes foliculares, la atresia folicular y la detención del desarrollo folicular en diferentes estados de desarrollo. Además los andrógenos en acción sinérgica con FSH tiende a oponerse a la atresia (13).

Se ha asumido que la hiperplasia de las células de la teca es el resultado de la estimulación crónica de LH y se asocia con excesiva producción de andrógenos. Estudios in vivo han demostrado evidencia convincente en favor de esta hipótesis. Células tecales aisladas de ovarios poliqu ísticos muestran una secreción preferencial de androstendiona con una alta sensibilidad a LH. EI exceso de andrógenos y el disturbio en la conversión de andrógenos a estrógenos con trastorno en los cambios cíclicos de estrógenos, responsable de los apropiados mecanismos de retroalimentación, son influídos por la constante producción de estrógenos extra glandulares. Así la 
excesiva secreción de andrógenos y la subsecuente conversión a estrógenos constituye la base para la instauración de la anovulación crónica (3-15).

Ahora bien en el hirsutismo idiopático la apariencia macroscópica de los ovarios es normal sin "estigmas" de quiste múltiple $\mathrm{ni}$ ovarios agrandados, pero con cápsula engrosada y sin signos de ovulación. Estudios recientes indican que un metabolito de testosterona, el $5 \alpha$ androstone - $3 \alpha, 17 \beta$ - diol, 3 glucoronido, se forma periféricamente, posiblemente por el folículo piloso y está elevado preferencialmente en las pacientes hirsutas.

Hiperandrogenismo de origen suprarrenal también está implicado en la etiología de sindromes similares al ovario poliqu ístico. Lo mismo sucede en la hiperplasia suprarrenal congénita del adulto. Evaluación endocrina revela un cuadro hormonal similar al descrito en ovario poliquístico con niveles basales elevados de $L H, T$ y $E_{1}$, consistentes con la conversión extraglândular de andrógenos a estrógenos.

En el estudio laparoscópico donde encontramos ovarios en diferentes estados morfológicos de respuesta al hiperandrogenismo, trastorno en la relación de gonadotropinas $\mathrm{y} / \mathrm{o}$ niveles de testosterona elevados, pensamos que corresponde a diferentes estados de evolución de un solo sindrome que hemos denominado de años atrás como el Sindrome de Ovario Androgénico (SOA) y que tiene una sola manifestación común que es el hiperandrogenismo primario o secundario del ovario y que puede comenzar por la anovulación crónica; manifestándose en algunos casos con predominancia de hirsutismo idiopático, produciendo cambios morfológicos con poliquistosis ovárica, hipertecosis tecal y por último, manifestarse en el clásico sindrome descrito por Stein y Leventhal en 1935. (Fig. 1).

Con relación a los niveles de testosterona total en pacientes con laparoscopias características de SOA en el $74 \%$ los
Figura No. 1

\section{HIPOTESIS EXPLICANDO EL S.O.A.}

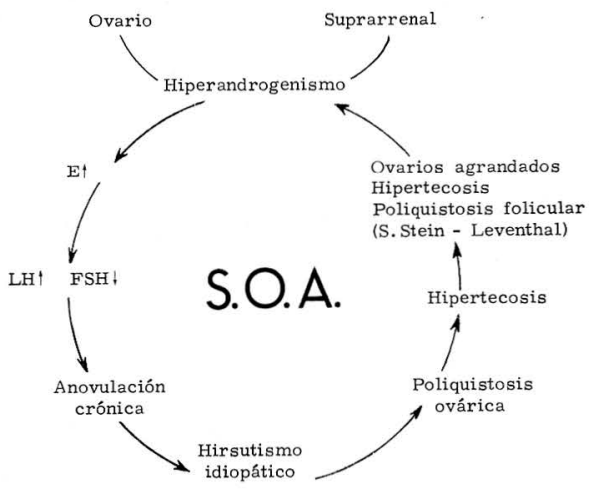

Figura No. 2

TESTOSTERONA EN PACIENTES CON LAPAROSCOPIA

"CARACTERISTICA" S.O.A.

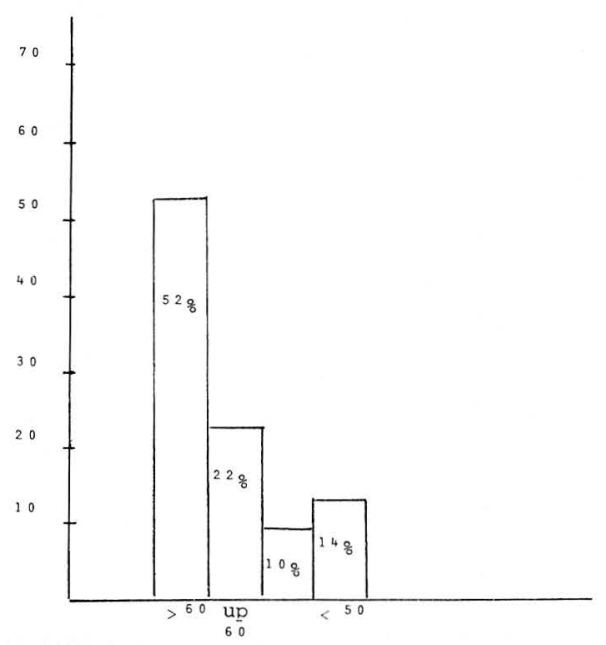


valores son $\geqslant 60 \mathrm{ng} / \mathrm{dl}$ (Figura No. 2) y en los de apariencia "dudosa" estos valores son de $70 \%$ (Figura No. 3), lo cual indica hiperandrogenismo similar en ambos grupos.

Figura No. 3

TESTOSTERONA EN PACIENTES CON

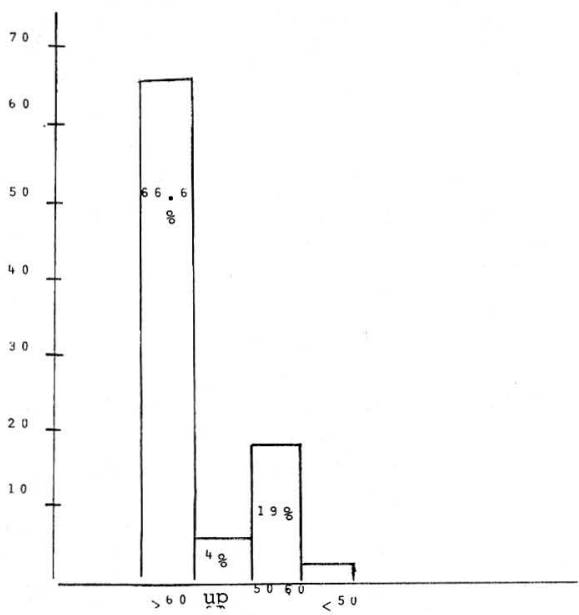

\section{BIBLIOGRAFIA}

1. STEIN, I.E., LEVENTHAL, M.L. Amenorrhea associated with bilateral policystic ovary. Am. J. Obstet. Gynecol. 29:181, 1935.

2. KIRSCHNER, M.A., I.R. ZUCHER and J. IRESPERSEN. Idiopathic hirsutism, an ovarian abnormality. N. Engl. J. Med. 294: 637, 1976.

3. REBAR, R., H.L. JUDD, S.S. YEN, J. RAKOFF, VANDERBER and NATTOLIN. Charecterization of the inappropiate gonadotropin secretion in PCO. J. Clin. Invest. 57: 1320, 1976.

4. GREENBLATT, P.B. and V.B. MAHESH. The Androgenic polycistic ovary. Am. J. Obstet. Gynecol. 125:712, 1976.

5. DE VONE, G., CZEKALA, H.L. JUDD and S.S. YEN. Circulating gonadotroping, estro- gens and androgens in polycistic ovarian disease. Am. J. Obstet. Gynecol. 121:496, 1975.

6. GOLDZIEHER, J.W. Polycistic ovarian disease. Fertil. Steril 35:371, 1981.

7. FRAENKEL, L. Thecoma and hyperthecosis of the ovary. J. Clin. Endocrin. Metab. 3:557, 1943.

8. ABRAHAM, G.E. and BUSTER, J.E. Peripheral and ovarian steroids in ovarian hyperthecosis. Obstet. Gynecol. 47:581, 1976.

9. LACHELIN, G.C. and YEN, S.S.C. Hypothalamic cronic anovulation. Am. J. Obstet. Gynecol. 130:825, 1978.

10. PAULSON, J., KELLER, L. WREST, W., WARREN, J.C. Free testosterone concentration in serum: Elevation is the hall mark of Hirsutism. Am. J. Obstet. Gynecol. 128:851, 1977.

11. GREEMBLATT, V., MAHESH. The androgenic polycistic ovary. Am. J. Obstet. Gynecol. 125:712, 1976.

12. LOUVET, J.P., HARMAN, S.M., SCHREIBER, J.R., ROSS, G.T. Evidence for role of androgen in folicular maturation. Endocrinology 97:366, 1975.

13. NIMROL, A., LINDNER, H.R. A synergetic effect of androgen on the stimulation of progesterone secretion by $\mathrm{FSH}$ in cultured rat granulosa cells. Mol. Cell. Endocrinol. 5: 315, 1976.

14. ERICKSON, G.F., HSUEH, A.J., QUIGLEY, M.E., REBAR, R.W. and YEN, S.S.C. Functional studies of aromatase activity in human granulosa cells from normal and POC. JCEM 49:514, 1979.

15. BAIRD, D.T., CORKER, C.S., DAVIDSON, N.W., HUNTER, W.M., NICHI E.A. and VANLOOK. Pituitary-ovarian relationships in polycistic ovary syndrome. J. Clin. Endocrinol. Metab. 45:798, 1977.

16. GOMEZ, G., ZULUAGA, A.L. Síndrome de Ovario Androgénico. Rev. Col. Obst. Gin. 31:219, 1980.

17. SMALS, A.G.H., KOPPENBERG, P.W.C. and BENRAAD, T.J. Plasma testosterone profiles in cushing's syndromes. J. Clin. Endocrinol. Metab. 45:240, 1977. 